\title{
Scattering Amplitudes and Conservative Binary Dynamics at $\mathcal{O}\left(G^{4}\right)$
}

\author{
Zvi Bern, ${ }^{1}$ Julio Parra-Martinez $\odot,{ }^{2}$ Radu Roiban, ${ }^{3}$ Michael S. Ruf $\odot,{ }^{4}$ \\ Chia-Hsien Shen $\odot,{ }^{5}$ Mikhail P. Solon, ${ }^{1}$ and Mao Zeng ${ }^{6}$ \\ ${ }^{1}$ Mani L. Bhaumik Institute for Theoretical Physics, University of California at Los Angeles, \\ Los Angeles, California 90095, USA \\ ${ }^{2}$ Walter Burke Institute for Theoretical Physics, California Institute of Technology, Pasadena, California 91125, USA \\ ${ }^{3}$ Institute for Gravitation and the Cosmos, Pennsylvania State University, University Park, Pennsylvania 16802, USA \\ ${ }^{4}$ Physikalisches Institut, Albert-Ludwigs-Universität Freiburg, Hermann-Herder-Strasse 3, 79104 Freiburg, Germany \\ ${ }^{5}$ Department of Physics, University of California at San Diego, 9500 Gilman Drive, La Jolla, California 92093-0319, USA \\ ${ }^{6}$ Rudolf Peierls Centre for Theoretical Physics, University of Oxford, Parks Road, Oxford OX1 3PU, United Kingdom
}

(Received 4 February 2021; accepted 11 March 2021; published 26 April 2021)

\begin{abstract}
Using scattering amplitudes, we obtain the potential contributions to conservative binary dynamics in general relativity at fourth post-Minkowskian order $\mathcal{O}\left(G^{4}\right)$. As in previous lower-order calculations, we harness powerful tools from the modern scattering amplitudes program including generalized unitarity, the double copy, and advanced multiloop integration methods, in combination with effective field theory. The classical amplitude involves polylogarithms with up to transcendental weight two and elliptic integrals. We derive the radial action directly from the amplitude, and determine the corresponding Hamiltonian in isotropic gauge. Our results are in agreement with known overlapping terms up to sixth post-Newtonian order, and with the probe limit. We also determine the post-Minkowskian energy loss from radiation emission at $\mathcal{O}\left(G^{3}\right)$ via its relation to the tail effect.
\end{abstract}

DOI: 10.1103/PhysRevLett.126.171601

Introduction.-The emergence of gravitational-wave science [1] has dramatically underscored the scientific value of observing the Universe through an entirely new lens, and will continue to fundamentally transform key areas in astronomy, cosmology, and particle physics. This calls for invigorating the theoretical framework necessary for interpreting signals at current and future detectors [2], and has thus galvanized new work in this direction. This includes a new program [3-5] for understanding the nature of gravitational-wave sources based on tools from scattering amplitudes and effective field theory (EFT). The connection of scattering amplitudes to general relativity corrections to Newton's potential has long been known [6-8]. Starting from foundational ideas from EFT applied to gravitationalwave physics [9], this new effort has integrated generalized unitarity [10], double-copy relations between gauge and gravity theories [11,12], EFT [3,8], and advanced multiloop integration [13-15]. These ideas culminated with advancing the state of the art by obtaining the $\mathcal{O}\left(G^{3}\right)$ conservative Hamiltonian for spinless compact binaries [4,5], whose various aspects have now been confirmed in multiple studies

Published by the American Physical Society under the terms of the Creative Commons Attribution 4.0 International license. Further distribution of this work must maintain attribution to the author(s) and the published article's title, journal citation, and DOI. Funded by SCOAP.
[16-20]. In this Letter, we extend previous methods and take the next step to obtain the contributions to conservative binary dynamics at $\mathcal{O}\left(G^{4}\right)$.

We focus here on the inspiral phase of conservative binary dynamics. Traditionally, this is approached using effective one-body [21], numerical relativity [22], gravitational self-force $[23,24]$, and perturbation theory in the post-Newtonian (PN) [25-28], post-Minkowskian (PM) [29,30], and nonrelativistic general relativity (NRGR) [9,31] frameworks. The PM approach has recently risen in prominence due to increased analytic control $[3-5,30,32-36]$. We work in this context given the natural fit with relativistic amplitudes.

The new amplitudes-based approach has, of course, benefited immensely from traditional methods, both in guidance and for confirming calculations. In turn, it has revealed connections between scattering amplitudes, classical observables, and gravitational self-force, inspiring new methods for obtaining perturbative corrections [19,20,36-39].

In this work we develop a new way to combine the techniques of Refs. [3-5], with advanced loop integration methods for classical integrals introduced in Ref. [40], and a link between the gauge-invariant amplitude and classical radial action, which further streamline the amplitudes approach and demonstrate its scalability. We derive the scattering amplitude in the classical limit for two massive scalars interacting via potential gravitons at $\mathcal{O}\left(G^{4}\right)$ and all orders in velocity. We also obtain the corresponding 
conservative two-body Hamiltonian and radial action, and determine the energy loss due to graviton emission at $\mathcal{O}\left(G^{3}\right)$ through its relation $[28,39,41]$ to the $\mathcal{O}\left(G^{4}\right)$ tail effect $[42,43]$. This order in perturbation theory presents new features from the tail effect, which manifests an infrared (IR) divergence due to the overlap between the momentum regions [44] of potential and radiation gravitons [45]. We also encounter a class of elliptic integrals, which complicates the analysis.

Scattering amplitudes are independent of gauge or coordinate choices, while EFT exposes universality in physical systems. These features greatly help identify emergent structures that can enhance our understanding of basic phenomena and lead to new tools that will further the cycle of innovation. Here, we present a remarkably simple gauge-invariant relation between the conservative scattering amplitude and the radial action based on a reorganization of the amplitude into classical and iteration pieces, distinct from that of Refs. [3-5,36]. It is well known that the radial action is also gauge invariant and encodes the dynamics of both bound and unbound orbits (see, e.g., Refs. [36,39,46]).

Classical dynamics from scattering amplitudes.-We focus on conservative two-body dynamics for spinless compact objects, described by the four-point amplitude $\mathcal{M}(\boldsymbol{q})$ of gravitationally interacting minimally coupled massive scalars. The two incoming particles of momenta $p_{1}, p_{2}$ have masses $m_{1}, m_{2}$, and we define $\sigma \equiv p_{1}$. $p_{2} / m_{1} m_{2}$ in mostly minus signature. We work in the center-of-mass (COM) frame where the momentum transfer $q^{\mu}=(0, \boldsymbol{q})$ is purely spatial. Following Refs. [40,47], we decompose $p_{1}, p_{2}$ into components orthogonal and along $q$, i.e., $p_{1}=\bar{p}_{1}-q / 2, p_{2}=\bar{p}_{2}+q / 2$ with $\bar{p}_{i} q=0$.

As described in Refs. [3-5], major simplifications are obtained by taking the classical limit early at the level of the integrand. This is achieved by an expansion in large angular momentum $J \gg \hbar$. We implement this by rescaling $q$ $\ell \rightarrow \lambda q, \lambda \ell$, where $\ell$ is any graviton momentum, and then expanding in small $\lambda$.

The classical limit therefore identifies the soft region, defined by the loop momentum scaling $\ell^{\mu}=(\omega, \ell) \sim$ $(\lambda, \lambda)$, as encoding classical dynamics. In the spirit of EFT [9], we simplify the analysis, especially in the presence of the tail effect at $\mathcal{O}\left(G^{4}\right)$, by focusing on the potential and (ultrasoft) radiation subregions defined by the scalings $\sim(v \lambda, \lambda)$ and $\sim(v \lambda, v \lambda)$, respectively. Here and below we use $v$ to denote the typical velocity of the binary constituents, corresponding to the small velocity that defines the PN expansion.

In the present work, we focus on the conservative part described by the potential contribution, and do not include radiation. This is sufficient for completely specifying the conservative dynamics through $\mathcal{O}\left(G^{3}\right)$ [4,5]. However, at $\mathcal{O}\left(G^{4}\right)$, radiative effects contribute to conservative dynamics via the tail effect [42]. Since the potential and radiation contributions overlap, this introduces scheme dependence and IR divergence [45]. We use conventional dimensional regularization, where the amplitudes, including graviton polarizations, are uniformly continued into $D=4-2 \epsilon$ dimensions.

Amplitude-action relation.-Conservative binary dynamics is fully encoded in the four-point amplitude $\mathcal{M}(\boldsymbol{q})$, truncated to the classical order. There exists another scalar gauge-invariant function which encodes the same dynamics, namely the radial action, which is defined as the integral of the radial momentum $p_{r}$ along the scattering trajectory, $I_{r}(J) \equiv \int p_{r} d r$, with appropriate regularization of the long-distance contribution. Here, we present a simple relation between these two gauge-invariant quantities, exposed through the EFT introduced in Ref. [3].

In the classical limit, the amplitude at $\mathcal{O}\left(G^{n}\right)$ contains a classical contribution that scales as $\lambda^{n-3}$ and iteration contributions that scale as $\lambda^{n-2}, \lambda^{n-1}, \ldots, \lambda^{-2}$. The latter correspond to iterations of lower-order amplitudes, are IR divergent, and cancel in physical observables. Although the full amplitude is invariant, the choice of pole structure of the iterations is not unique and the classical part is modified accordingly. Previously this was chosen to align with the matter energy poles in the EFT for direct cancellation without explicit evaluation [3]. This choice also revealed a connection between the classical amplitude and the local $\mathrm{COM}$ momentum in isotropic gauge, first observed in $[4,5]$ and later proven in $[32,36]$.

In the present analysis, we instead expand the matter poles about the momentum component along $\hat{z}$, the direction of the spatial component of $\bar{p}_{1}$. Inspired by the eikonal approximation [48], this prescription reveals a gauge-invariant "amplitude-action relation"

$$
i \mathcal{M}(\boldsymbol{q})=\int_{J}\left(e^{i I_{r}(J)}-1\right)
$$

between the amplitude $\mathcal{M}(\boldsymbol{q})$ and the radial action $I_{r}(J)$. The classical part of the amplitude then corresponds to the term linear in $I_{r}(J)$, given by

$$
\tilde{I}_{r}(\boldsymbol{q})=\int_{J} I_{r}(J) \equiv 4 E|\boldsymbol{p}| \int \mu^{-2 \epsilon} d^{D-2} \boldsymbol{b} e^{i \boldsymbol{q} \cdot \boldsymbol{b}} I_{r}(J),
$$

where $\boldsymbol{p}$ is the spatial momentum, $E$ is the total energy, $|\boldsymbol{b}|=J /|\boldsymbol{p}|$ is the impact parameter in the COM frame, and $\mu$ is the renormalization scale. As will be shown elsewhere [49], terms higher order in $I_{r}(J)$ in the relation (1) have the following structure under our prescription:

$$
\begin{aligned}
\int_{J} \frac{\left[i I_{r}(J)\right]^{n}}{n !} & =i \int_{\boldsymbol{\ell}} \frac{\tilde{I}_{r}\left(\boldsymbol{\ell}_{1}\right), \ldots, \tilde{I}_{r}\left(\boldsymbol{\ell}_{n}\right)}{Z_{1}, \ldots, Z_{n-1}} \\
Z_{j} & =-4 E|\boldsymbol{p}|\left[\left(\boldsymbol{\ell}_{\mathbf{1}}+\boldsymbol{\ell}_{\mathbf{2}}+\cdots+\boldsymbol{\ell}_{\boldsymbol{j}}\right) \cdot \hat{z}+i 0\right],
\end{aligned}
$$


where $\int_{\ell} \equiv \int \prod_{i=1}^{n}\left[d^{D-1} \boldsymbol{\ell}_{\boldsymbol{i}} /(2 \pi)^{D-1}\right](2 \pi)^{D-1} \delta\left(\sum_{j=1}^{n} \boldsymbol{\ell}_{\boldsymbol{j}}-\boldsymbol{q}\right)$ and we only keep the leading classical expansion in the numerator of Eq. (3). Crucially, we manifest the pole structure in $Z_{j}$ when computing the amplitude such that the classical part can be isolated and iterations can be safely dropped without explicitly evaluating them, following the path of Ref. [3]. With our prescription, we avoid tracking such terms, which is necessary in standard eikonal exponentiation [48].

This amplitude-action relation then dictates the iteration structure in the amplitude when expanded in $G$. To illustrate, Eqs. (1) and (3) with the expansions $\mathcal{M}(\boldsymbol{q})=$ $\sum_{n} \mathcal{M}_{n}(\boldsymbol{q})$ and $\tilde{I}_{r}(\boldsymbol{q})=\sum_{n} \tilde{I}_{r, n}(\boldsymbol{q})$ yield

$$
\begin{aligned}
& \mathcal{M}_{1}(\boldsymbol{q})=\tilde{I}_{r, 1}(\boldsymbol{q}), \\
& \mathcal{M}_{2}(\boldsymbol{q})=\tilde{I}_{r, 2}(\boldsymbol{q})+\int_{\boldsymbol{e}} \frac{\tilde{I}_{r, 1} \tilde{I}_{r, 1}}{Z_{1}}, \\
& \mathcal{M}_{3}(\boldsymbol{q})=\tilde{I}_{r, 3}(\boldsymbol{q})+\int_{\boldsymbol{e}} \frac{\tilde{I}_{r, 1}^{3}}{Z_{1} Z_{2}}+\int_{\boldsymbol{e}} \frac{\tilde{I}_{r, 1} \tilde{I}_{r, 2}}{Z_{1}},
\end{aligned}
$$

where the sum over permutations of distinct $\tilde{I}_{r, n}$ is implicit; for instance, $\tilde{I}_{r, 1} \tilde{I}_{r, 2} \equiv \tilde{I}_{r, 1}\left(\boldsymbol{\ell}_{\mathbf{1}}\right) \tilde{I}_{r, 2}\left(\boldsymbol{\ell}_{\mathbf{2}}\right)+\tilde{I}_{r, 2}\left(\boldsymbol{\ell}_{\mathbf{1}}\right) \tilde{I}_{r, 1}\left(\boldsymbol{\ell}_{\mathbf{2}}\right)$ while $\tilde{I}_{r, 1}^{3} \equiv \tilde{I}_{r, 1}\left(\boldsymbol{\ell}_{\mathbf{1}}\right) \tilde{I}_{r, 1}\left(\boldsymbol{\ell}_{\mathbf{2}}\right) \tilde{I}_{r, 1}\left(\boldsymbol{\ell}_{\mathbf{3}}\right)$. As we can see, the classical part of the amplitude with this pole choice is directly the radial action, in contrast to Refs. [3-5]. We have explicitly verified Eqs. (1) and (3) through $\mathcal{O}\left(G^{4}\right)$ by comparing the amplitude calculation in EFT to the radial action from classical mechanics [49]. With some care, the relation (1) can be established more generally to higher orders in $G$ [49].

Constructing the integrand.-The calculation of the amplitude through $\mathcal{O}\left(G^{4}\right)$ begins with the construction of the classical limit of the three-loop integrand. We use generalized unitarity, as described in Ref. [5], which builds gravitational loop integrands directly from on-shell tree amplitudes of scalars and gravitons. The maximal-cut version [50] of generalized unitarity adopted here organizes the cuts hierarchically.

As explained in Ref. [5], potential contributions come from cuts with at least one matter line per loop and with no gravitons beginning and ending on the same matter line. We drop contributions not of this type, such as self-energy loops and matter contact diagrams. The eight distinct contributing generalized cuts are shown in Fig. 1, with the others given by simple relabelings.

We use the $D$-dimensional tree-level BCJ double copy [12] to obtain the gravitational tree amplitudes from corresponding gauge-theory ones. The dilaton and antisymmetric tensor which naturally appear as intermediate states are straightforwardly eliminated by including graviton physical-state projectors on the cut legs. Conveniently, the reference light-cone momentum in their definition

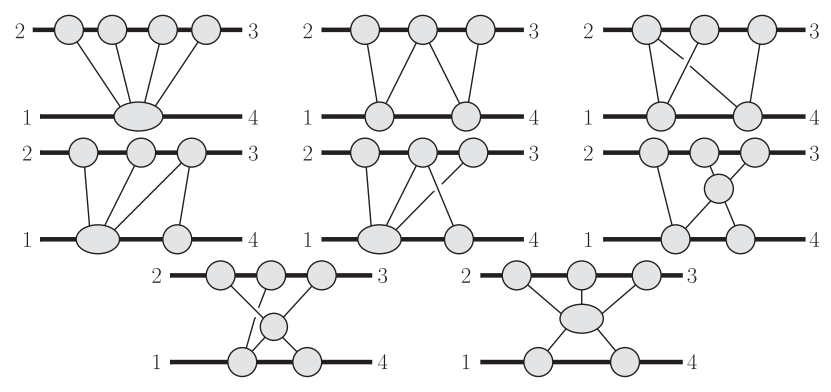

FIG. 1. Generalized unitarity cuts encoding potential-region contributions to binary dynamics. Ovals represent tree amplitudes while exposed lines depict on-shell states. Thin and thick lines denote gravitons and massive scalars, respectively.

cancels automatically, by organizing the tree amplitudes so that they obey generalized Ward identities, following Ref. [51]. A similar strategy was used earlier for simpler one- and two-loop calculations $[4,5,35]$.

The resulting integrand is then organized in terms of 51 distinct cubic-vertex Feynman-like diagrams, of which three are shown in Fig. 2. All remaining diagrams are obtained by relabeling the external momenta. The integrals are then further reduced to a basis using integration by parts [13] implemented through FIRE6 [15], and graph symmetries. We isolate iteration integrals, which cancel in the EFT matching prior to explicit integration.

Throughout, we work with rescaled variables $u_{i}=$ $\bar{p}_{i} /\left|\bar{p}_{i}\right|$, following Ref. [40], so that the integrals depend on the single variable $y=u_{1} \cdot u_{2}$, enormously simplifying the analysis. This also factors out the mass dependence, clearly exposing the overlap between PM gravity and gravitational self-force $[37,38,52,53]$.

Evaluating the integrals.-Our strategy for integration combines the nonrelativistic method of Refs. [3-5] and the method of differential equations following Ref. [40]. The two methods complement each other to systematically determine the analytic result to all orders in velocity, and have been applied to multiple examples [17,18,54-56].

The nonrelativistic method evaluates integrals as an expansion in powers of $v$, where the leading term serves as the boundary value for solving the differential equations. This also efficiently isolates the iterations in Eq. (3), which cancel directly in the EFT matching. The first step is to perform energy loop integration by localizing to residues
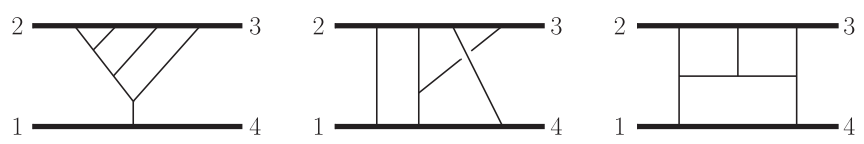

FIG. 2. Sample diagrams at $\mathcal{O}\left(G^{4}\right)$. From left to right: a contribution in the probe limit, a nonplanar diagram that contains iteration terms, and a diagram that contains contributions related to the tail effect. 
TABLE I. Functions specifying the amplitude in Eq. (6).

$$
\begin{aligned}
& h_{1}=\frac{1151-3336 \sigma+3148 \sigma^{2}-912 \sigma^{3}+339 \sigma^{4}-552 \sigma^{5}+210 \sigma^{6}}{12\left(\sigma^{2}-1\right)}, \\
& h_{2}=\frac{1}{2}\left(5-76 \sigma+150 \sigma^{2}-60 \sigma^{3}-35 \sigma^{4}\right) \text {, } \\
& h_{3}=\sigma \frac{\left(-3+2 \sigma^{2}\right)}{4\left(\sigma^{2}-1\right)}\left(11-30 \sigma^{2}+35 \sigma^{4}\right) \text {, } \\
& h_{4}=\frac{1}{144\left(\sigma^{2}-1\right)^{2} \sigma^{7}}\left(-45+207 \sigma^{2}-1471 \sigma^{4}+13349 \sigma^{6}-37566 \sigma^{7}+104753 \sigma^{8}-12312 \sigma^{9}-102759 \sigma^{10}\right. \\
& \left.-105498 \sigma^{11}+134745 \sigma^{12}+83844 \sigma^{13}-101979 \sigma^{14}+13644 \sigma^{15}+10800 \sigma^{16}\right), \\
& h_{5}=\frac{1}{4\left(\sigma^{2}-1\right)}\left(1759-4768 \sigma+3407 \sigma^{2}-1316 \sigma^{3}+957 \sigma^{4}-672 \sigma^{5}+341 \sigma^{6}+100 \sigma^{7}\right) \text {, } \\
& h_{6}=\frac{1}{24\left(\sigma^{2}-1\right)^{2}}\left(1237+7959 \sigma-25183 \sigma^{2}+12915 \sigma^{3}+18102 \sigma^{4}-12105 \sigma^{5}-9572 \sigma^{6}+2973 \sigma^{7}+5816 \sigma^{8}-2046 \sigma^{9}\right) \text {, } \\
& h_{7}=2 \sigma \frac{\left(-852-283 \sigma^{2}-140 \sigma^{4}+75 \sigma^{6}\right)}{3\left(\sigma^{2}-1\right)}, \\
& h_{8}=\frac{\sigma}{8\left(\sigma^{2}-1\right)^{2}}\left(-304-99 \sigma+672 \sigma^{2}+402 \sigma^{3}-192 \sigma^{4}-719 \sigma^{5}-416 \sigma^{6}+540 \sigma^{7}+240 \sigma^{8}-140 \sigma^{9}\right) \text {, } \\
& h_{9}=\frac{1}{2}\left(52-532 \sigma+351 \sigma^{2}-420 \sigma^{3}+30 \sigma^{4}-25 \sigma^{6}\right) \text {, } \\
& h_{10}=2\left(27+90 \sigma^{2}+35 \sigma^{4}\right) \text {, } \\
& h_{11}=20+111 \sigma^{2}+30 \sigma^{4}-25 \sigma^{6} \text {, } \\
& h_{12}=\frac{834+2095 \sigma+1200 \sigma^{2}}{2\left(\sigma^{2}-1\right)} \text {, } \\
& h_{13}=-\frac{1183+2929 \sigma+2660 \sigma^{2}+1200 \sigma^{3}}{2\left(\sigma^{2}-1\right)}, \\
& h_{14}=\frac{7\left(169+380 \sigma^{2}\right)}{4(\sigma-1)} \text {. }
\end{aligned}
$$

given by matter poles in the potential region. For a given integral $\mathcal{I}$ the result of energy integration is

$$
\int \prod_{i=1}^{3} \frac{d \omega_{i}}{2 \pi} \mathcal{I}\left(\omega_{1}, \omega_{2}, \omega_{3}\right)=\sum_{i} S_{i} \operatorname{Res}_{i} \mathcal{I}\left(\omega_{1}, \omega_{2}, \omega_{3}\right)
$$

where the sum runs over triplets of matter poles on which the residues are evaluated. The symmetry factors $S_{i}$ are determined from the cuts in Fig. 1, building upon the prescription in Ref. [5]. The remaining three-dimensional integrals are then expanded in $v$, and reduced to master integrals via integration by parts [13] using FIRE6 [15]. Iterations are identified by the pole structure in Eq. (3), and the final integrals are the same as in NRGR [9].

Following Ref. [40], we use differential equations to analytically solve integrals, or to obtain solutions expanded in $v$ to very high orders. The boundary conditions for the univariate differential equations are imposed in the leading PN expansion. A new feature at $\mathcal{O}\left(G^{4}\right)$ is the appearance of elliptic integrals, which precludes a canonical basis [57]. This complicates the structure of the differential equations. We solve part of the system exactly in terms of classical polylogarithms up to weight three and complete elliptic integrals. The remaining contribution to the amplitude is determined by constructing an ansatz as a linear combination of a subset of the known functions with rational coefficients, whose parameters we fix using a series solution to the differential equations up to $\mathcal{O}\left(v^{60}\right)$. In this way we obtain the full velocity dependence, which can be checked, for instance, by obtaining a series solution up to $\mathcal{O}\left(v^{400}\right)$, finding perfect agreement. Details will be presented in Ref. [49]. 
Amplitude.-Performing this calculation, we obtain the following 4PM classical amplitude in the potential region:

$$
\begin{aligned}
\mathcal{M}_{4}(\boldsymbol{q})= & G^{4} M^{7} \nu^{2}|\boldsymbol{q}|\left(\frac{\boldsymbol{q}^{2}}{4^{1 / 3} \tilde{\mu}^{2}}\right)^{-3 \epsilon} \pi^{2}\left[\mathcal{M}_{4}^{p}+\nu\left(\frac{\mathcal{M}_{4}^{t}}{\epsilon}+\mathcal{M}_{4}^{f}\right)\right]+\int_{\ell} \frac{\tilde{I}_{r, 1}^{4}}{Z_{1} Z_{2} Z_{3}}+\int_{\ell} \frac{\tilde{I}_{r, 1}^{2} \tilde{I}_{r, 2}}{Z_{1} Z_{2}}+\int_{\ell} \frac{\tilde{I}_{r, 1} \tilde{I}_{r, 3}}{Z_{1}}+\int_{\ell} \frac{\tilde{I}_{r, 2}^{2}}{Z_{1}}, \\
\mathcal{M}_{4}^{p}= & -\frac{35\left(1-18 \sigma^{2}+33 \sigma^{4}\right)}{8\left(\sigma^{2}-1\right)}, \quad \mathcal{M}_{4}^{t}=h_{1}+h_{2} \log \left(\frac{\sigma+1}{2}\right)+h_{3} \frac{\operatorname{arccosh}(\sigma)}{\sqrt{\sigma^{2}-1}} \\
\mathcal{M}_{4}^{f}= & h_{4}+h_{5} \log \left(\frac{\sigma+1}{2}\right)+h_{6} \frac{\operatorname{arccosh}(\sigma)}{\sqrt{\sigma^{2}-1}}+h_{7} \log (\sigma)-h_{2} \frac{2 \pi^{2}}{3}+h_{8} \frac{\operatorname{arccosh}^{2}(\sigma)}{\sigma^{2}-1}+h_{9}\left[\operatorname{Li}_{2}\left(\frac{1-\sigma}{2}\right)+\frac{1}{2} \log ^{2}\left(\frac{\sigma+1}{2}\right)\right] \\
& +h_{10}\left[\operatorname{Li}_{2}\left(\frac{1-\sigma}{2}\right)-\frac{\pi^{2}}{6}\right]+h_{11}\left[\operatorname{Li}_{2}\left(\frac{1-\sigma}{1+\sigma}\right)-\operatorname{Li}_{2}\left(\frac{\sigma-1}{\sigma+1}\right)+\frac{\pi^{2}}{3}\right]+h_{2} \frac{2 \sigma\left(2 \sigma^{2}-3\right)}{\left(\sigma^{2}-1\right)^{3 / 2}}\left[\operatorname{Li}_{2}\left(\sqrt{\frac{\sigma-1}{\sigma+1}}\right)-\operatorname{Li}_{2}\left(-\sqrt{\frac{\sigma-1}{\sigma+1}}\right)\right] \\
& +\frac{2 h_{3}}{\sqrt{\sigma^{2}-1}}\left[\operatorname{Li}_{2}\left(1-\sigma-\sqrt{\sigma^{2}-1}\right)-\operatorname{Li}_{2}\left(1-\sigma+\sqrt{\sigma^{2}-1}\right)+5 \operatorname{Li}_{2}\left(\sqrt{\frac{\sigma-1}{\sigma+1}}\right)-5 \operatorname{Li}_{2}\left(-\sqrt{\frac{\sigma-1}{\sigma+1}}\right)\right. \\
& \left.+2 \log \left(\frac{\sigma+1}{2}\right) \operatorname{arccosh}(\sigma)\right]+h_{12} K^{2}\left(\frac{\sigma-1}{\sigma+1}\right)+h_{13} K\left(\frac{\sigma-1}{\sigma+1}\right) E\left(\frac{\sigma-1}{\sigma+1}\right)+h_{14} E^{2}\left(\frac{\sigma-1}{\sigma+1}\right)
\end{aligned}
$$

where $M=m_{1}+m_{2}$ is the total mass, $\nu=m_{1} m_{2} / M^{2}$ is the symmetric mass ratio, $\tilde{\mu}^{2}=4 \pi \mu^{2} e^{-\gamma_{E}}$ is the renormalization scale in $\overline{\mathrm{MS}}$ scheme. $\mathrm{Li}_{2}$ is the dilogarithm, and $K$ and $E$ are the complete elliptic integrals of the first and second kind, respectively. The coefficient functions $h_{i}$ are collected in Table I.

We emphasize that Eq. (6) uses dimensional regularization with $D=4-2 \epsilon$ and that $\tilde{I}_{r, 1}, \tilde{I}_{r, 2}$, and $\tilde{I}_{r, 3}$ are expanded to the classical limit. The tail effect manifests as a $1 / \epsilon$ IR divergence in the classical term, due to the overlap between potential and radiation contributions [45]. Including the latter, which is not done here, would cancel this divergence and the associated scheme dependence, replace $\tilde{\mu}$ with a physical scale, and also add finite terms. Note that the scheme dependence starts at 4PN and enters only through the coefficient functions $h_{4}, h_{5}$, and $h_{6}$.

The amplitude naturally exposes the simple dependence in the symmetric mass ratio $\nu$, consistent with Ref. [37]. The leading term $\mathcal{M}_{4}^{p}$ agrees with the result obtained using the Schwarzschild solution [58,59]. The next-to-leading terms $\mathcal{M}_{4}^{t}$ and $\mathcal{M}_{4}^{f}$ overlap with first-order self-force [37].

As for the $\mathcal{O}\left(G^{3}\right)$ case, the ultrarelativistic limit of the conservative result in Eq. (6) does not smoothly match onto the massless case. The amplitude has a leading power discontinuity of the form $\sim G^{4} \boldsymbol{p}^{8}|\boldsymbol{q}|\left(m_{1}+m_{2}\right) /\left(m_{1} m_{2}\right)$, consistent with dimensional analysis. One can expect this to cancel with radiative effects $[56,60]$.

Given the relation in Eq. (1), it is straightforward to derive the radial action from the classical term in Eq. (6) via inverting Eq. (2)

$$
\begin{aligned}
I_{r, 4}(J)= & -\frac{G^{4} M^{7} \nu^{2} \pi p^{2}}{8 E J^{3}}\left(\frac{4 \tilde{\mu}^{2} e^{2 \gamma_{E}} J^{2}}{p^{2}}\right)^{4 \epsilon} \\
& \times\left[\mathcal{M}_{4}^{p}+\nu\left(\frac{\mathcal{M}_{4}^{t}}{\epsilon}+\mathcal{M}_{4}^{f}-14 \mathcal{M}_{4}^{t}\right)\right],
\end{aligned}
$$

which inherits the simple mass dependence from the amplitude $[19,37]$. Moreover, we have checked that $I_{r, 1}$, $I_{r, 2}$, and $I_{r, 3}$ obtained from the iteration terms in Eq. (6) are consistent with the known results [36].

The scattering angle is then given by $\chi=-\partial I_{r} / \partial J$. We compare to the $\mathcal{O}\left(G^{4}\right)$ scattering angle obtained from potential contributions to the Hamiltonian up to 5PN, given in Eqs. (21)-(26) of [61] and Eq. (5) of [62]. We find agreement including terms that depend on conventional dimensional regularization, which first enter at 4PN. (Since this work first appeared the authors have extended their calculation to 6PN [63], finding agreement with our results.) We also compare the regularization-scheme-independent $\pi^{3}$ terms with the 6PN result in Eq. (8.4) of Ref. [20], and find agreement. Here, we have only focused on potential-region contributions to the dynamics. A comparison with the full 4PN Hamiltonian [26,27] and remaining $5 \mathrm{PN}$ and $6 \mathrm{PN}$ parts of the scattering angle $[19,20,39]$ would require radiation contributions, which we have not pursued here.

As discussed in Refs. [28,39,41], the tail term $\mathcal{M}_{4}^{t}$ is related to the energy loss $\Delta E$ from radiation emission, and we thus identify

$$
\Delta E=\frac{G^{3} M^{7} \nu^{3} \pi p^{2}}{4 E^{2} J^{3}} \mathcal{M}_{4}^{t},
$$

in the COM frame. We have compared this to the direct calculation of the energy loss in Ref. [64] using the formalism of Ref. [34], finding agreement. Additional checks of $\Delta E$ are discussed in Ref. [64]. We can also obtain other observables for bound orbits via analytic continuation [36]; details will be presented elsewhere [49].

Hamiltonian.-Following the approach in Ref. [3], we can construct the two-body Hamiltonian in isotropic gauge 


$$
H^{\text {iso }}=E_{1}+E_{2}+\sum_{n=1}^{\infty} \frac{G^{n}\left(r^{2} \tilde{\mu}^{2} e^{2 \gamma_{E}}\right)^{n \epsilon}}{r^{n}} c_{n}\left(\boldsymbol{p}^{2}\right)
$$

where $r$ is the distance between bodies, and $E_{i}=$ $\sqrt{\boldsymbol{p}^{2}+m_{i}^{2}}$ are the energies of the incoming particles.
The $\mathcal{O}(\epsilon)$ corrections are relevant when $c_{n}\left(\boldsymbol{p}^{2}\right)$ is divergent, which first occurs at $\mathcal{O}\left(G^{4}\right)$ in isotropic gauge. The coefficients $c_{n}\left(\boldsymbol{p}^{2}\right)$ are determined by matching as in Refs. [3-5], but using the new pole choice in Eq. (3). Upon accounting for this we find

$$
\begin{aligned}
c_{4}= & \frac{M^{7} \nu^{2}}{4 \xi E^{2}}\left[\mathcal{M}_{4}^{p}+\nu\left(\frac{\mathcal{M}_{4}^{t}}{\epsilon}+\mathcal{M}_{4}^{f}-10 \mathcal{M}_{4}^{t}\right)\right]+\mathcal{D}^{3}\left[\frac{E^{3} \xi^{3}}{3} c_{1}^{4}\right]+\mathcal{D}^{2}\left[\left(\frac{E^{3} \xi^{3}}{\boldsymbol{p}^{2}}+\frac{E \xi(3 \xi-1)}{2}\right) c_{1}^{4}-2 E^{2} \xi^{2} c_{1}^{2} c_{2}\right] \\
& +\left(\mathcal{D}+\frac{1}{\boldsymbol{p}^{2}}\right)\left[E \xi\left(2 c_{1} c_{3}+c_{2}^{2}\right)+\left(\frac{4 \xi-1}{4 E}+\frac{2 E^{3} \xi^{3}}{\boldsymbol{p}^{4}}+\frac{E \xi(3 \xi-1)}{\boldsymbol{p}^{2}}\right) c_{1}^{4}+\left((1-3 \xi)-\frac{4 E^{2} \xi^{2}}{\boldsymbol{p}^{2}}\right) c_{1}^{2} c_{2}\right],
\end{aligned}
$$

where $\xi=E_{1} E_{2} / E^{2}$, and $\mathcal{D}=d / d \boldsymbol{p}^{2}$ denotes differentiation with respect to $\boldsymbol{p}^{2}$. The lower-order coefficients $c_{1}, c_{2}$, and $c_{3}$ can be found in Eq. (10) of Ref. [4]. The final explicit result for $c_{4}$ is included in the ancillary file [65]. Note that the iteration terms in Eq. (6) cancel in this matching, providing another nontrivial check.

Conclusions. - In this Letter we applied and extended amplitudes and EFT-based methods to determine the potential contribution to binary dynamics at $\mathcal{O}\left(G^{4}\right)$, offering a first look at the PM tail effect. Radiation contributions need to be added to completely determine the $\mathcal{O}\left(G^{4}\right)$ dynamics. It would be important to investigate the application of our methods to this problem. It would also be interesting to study closed-orbit observables at $\mathcal{O}\left(G^{4}\right)$, as was done for $\mathcal{O}\left(G^{3}\right)$ [53], and via analytic continuation [36]. The interplay with gravitational self-force $[23,24,37,38,52,66]$, the structure of long-distance logarithms at higher orders via renormalization group techniques [41,43,67], and the complete tail contribution in the PM framework also deserve further study.

Aside from the obvious application to gravitational-wave physics, our calculation elucidates emerging structures and identifies new tools. The amplitude-action relation in Eq. (1) greatly clarifies the link between scattering amplitudes and classical mechanics. It is natural to expect that this structure holds more generally. Obvious extensions within the PM framework include spin $[52,54,68]$, tidal $[55,59,69]$, and radiation [70,71] effects. Most excitingly, the methods applied here are not close to being exhausted.

We thank Samuel Abreu, Johannes Blümlein, Alessandra Buonanno, Clifford Cheung, Thibault Damour, Lance Dixon, Enrico Herrmann, Andrés Luna, Rafael Porto, Ira Rothstein, Jan Steinhoff, Gabriele Veneziano, and Justin Vines for helpful discussions. Z.B. is supported by the U.S. Department of Energy (DOE) under Award No. DE-SC0009937. J. P.-M. is supported by the U.S. Department of Energy (DOE) under Award No. DE-SC0011632. R. R. is supported by the U.S. Department of Energy (DOE) under Grant No. DESC0013699. M. S. R.'s work is funded by the German
Research Foundation (DFG) within the Research Training Group GRK 2044. C.-H.S. is supported by the U.S. Department of Energy (DOE) under Award No. DESC0009919. M.P.S. is supported by the David Saxon Presidential Term Chair. M.Z.'s work is supported by the U.K. Royal Society through Grant No. URF\R1\20109. We thank the Mani L. Bhaumik Institute for support.

[1] B. P. Abbott et al. (LIGO Scientific and Virgo Collaborations), Phys. Rev. Lett. 116, 061102 (2016); 119, 161101 (2017).

[2] M. Punturo et al. Classical Quantum Gravity 27, 194002 (2010); P. Amaro-Seoane et al. (LISA Collaboration), arXiv:1702.00786; D. Reitze et al., Bull. Am. Astron. Soc. 51, 035 (2019), https://baas.aas.org/pub/2020n7i035.

[3] C. Cheung, I. Z. Rothstein, and M. P. Solon, Phys. Rev. Lett. 121, 251101 (2018).

[4] Z. Bern, C. Cheung, R. Roiban, C. H. Shen, M. P. Solon, and M. Zeng, Phys. Rev. Lett. 122, 201603 (2019).

[5] Z. Bern, C. Cheung, R. Roiban, C. H. Shen, M. P. Solon, and M. Zeng, J. High Energy Phys. 10 (2019) 206.

[6] Y. Iwasaki, Prog. Theor. Phys. 46, 1587 (1971); Lett. Nuovo Cimento 1, 783 (1971); S. N. Gupta and S. F. Radford, Phys. Rev. D 19, 1065 (1979).

[7] J. F. Donoghue, Phys. Rev. D 50, 3874 (1994); N. E. J. Bjerrum-Bohr, J. F. Donoghue, and B. R. Holstein, Phys. Rev. D 67, 084033 (2003); 71, 069903(E) (2005); N. E. J. Bjerrum-Bohr, J. F. Donoghue, and P. Vanhove, J. High Energy Phys. 02 (2014) 111.

[8] D. Neill and I. Z. Rothstein, Nucl. Phys. B877, 177 (2013).

[9] W. D. Goldberger and I. Z. Rothstein, Phys. Rev. D 73, 104029 (2006).

[10] Z. Bern, L. J. Dixon, D. C. Dunbar, and D. A. Kosower, Nucl. Phys. B425, 217 (1994); B435, 59 (1995); Z. Bern and A. G. Morgan, Nucl. Phys. B467, 479 (1996); Z. Bern, L. J. Dixon, and D. A. Kosower, Nucl. Phys. B513, 3 (1998); R. Britto, F. Cachazo, and B. Feng, Nucl. Phys. B725, 275 (2005).

[11] H. Kawai, D. C. Lewellen, and S. H. H. Tye, Nucl. Phys. B269, 1 (1986); Z. Bern, L. J. Dixon, M. Perelstein, and J. S. Rozowsky, Nucl. Phys. B546, 423 (1999). 
[12] Z. Bern, J. J. M. Carrasco, and H. Johansson, Phys. Rev. D 78, 085011 (2008); Phys. Rev. Lett. 105, 061602 (2010); Z. Bern, J. J. M. Carrasco, W. M. Chen, H. Johansson, R. Roiban, and M. Zeng, Phys. Rev. D 96, 126012 (2017); Z. Bern, J. J. Carrasco, W. M. Chen, A. Edison, H. Johansson, J. Parra-Martinez, R. Roiban, and M. Zeng, Phys. Rev. D 98, 086021 (2018); Z. Bern, J. J. Carrasco, M. Chiodaroli, H. Johansson, and R. Roiban, arXiv:1909.01358.

[13] K. G. Chetyrkin and F. V. Tkachov, Nucl. Phys. B192, 159 (1981); S. Laporta, Int. J. Mod. Phys. A 15, 5087 (2000).

[14] A. V. Kotikov, Phys. Lett. B 254, 158 (1991); Z. Bern, L. J. Dixon, and D. A. Kosower, Nucl. Phys. B412, 751 (1994); E. Remiddi, Nuovo Cimento A 110, 1435 (1997); T. Gehrmann and E. Remiddi, Nucl. Phys. B580, 485 (2000).

[15] A. V. Smirnov, J. High Energy Phys. 10 (2008) 107; A. V. Smirnov and F. S. Chuharev, Comput. Phys. Commun. 247, 106877 (2020).

[16] J. Blümlein, A. Maier, P. Marquard, and G. Schäfer, Phys. Lett. B 807, 135496 (2020).

[17] C. Cheung and M. P. Solon, J. High Energy Phys. 06 (2020) 144.

[18] G. Kälin, Z. Liu, and R. A. Porto, Phys. Rev. Lett. 125, 261103 (2020).

[19] D. Bini, T. Damour, and A. Geralico, Phys. Rev. D 102, 024062 (2020).

[20] D. Bini, T. Damour, and A. Geralico, Phys. Rev. D 102, 024061 (2020).

[21] A. Buonanno and T. Damour, Phys. Rev. D 59, 084006 (1999); 62, 064015 (2000).

[22] F. Pretorius, Phys. Rev. Lett. 95, 121101 (2005); M. Campanelli, C. O. Lousto, P. Marronetti, and Y. Zlochower, Phys. Rev. Lett. 96, 111101 (2006); J. G. Baker, J. Centrella, D. I. Choi, M. Koppitz, and J. van Meter, Phys. Rev. Lett. 96, 111102 (2006).

[23] Y. Mino, M. Sasaki, and T. Tanaka, Phys. Rev. D 55, 3457 (1997); T. C. Quinn and R. M. Wald, Phys. Rev. D 56, 3381 (1997).

[24] E. Poisson, A. Pound, and I. Vega, Living Rev. Relativity 14, 7 (2011); L. Barack and A. Pound, Rep. Prog. Phys. 82, 016904 (2019).

[25] J. Droste, Proc. Acad. Sci. Amsterdam 19, 447 (1916); H. A. Lorentz and J. Droste, K. Akad. Wet. Amsterdam 26, 392 (1917); English translation in "Lorentz Collected Papers," edited by P. Zeeman and A. D. Fokker (1934-1939), Vol. 5, p. 330; A. Einstein, L. Infeld, and B. Hoffmann, Ann. Math. 39, 65 (1938); T. Ohta, H. Okamura, T. Kimura, and K. Hiida, Prog. Theor. Phys. 50, 492 (1973); T. Damour and N. Deruelle, Phys. Lett. 87A, 81 (1981); T. Damour, C. R. Acad. Sci. Paris, Sér. II 294, 1355 (1982); P. Jaranowski and G. Schäfer, Phys. Rev. D 57, 7274 (1998); 63, 029902(E) (2000); T. Damour, P. Jaranowski, and G. Schäfer, Phys. Rev. D 62, 044024 (2000); L. Blanchet and G. Faye, Phys. Lett. A 271, 58 (2000); Phys. Rev. D 63, 062005 (2001); V. C. de Andrade, L. Blanchet, and G. Faye, Classical Quantum Gravity 18, 753 (2001); T. Damour, P. Jaranowski, and G. Schäfer, Phys. Lett. B 513, 147 (2001); L. Blanchet and B. R. Iyer, Classical Quantum Gravity 20, 755 (2003); L. Blanchet, T. Damour, and G. Esposito-Farese, Phys. Rev. D 69, 124007 (2004).
[26] T. Damour, P. Jaranowski, and G. Schäfer, Phys. Rev. D 89, 064058 (2014); P. Jaranowski and G. Schäfer, Phys. Rev. D 92, 124043 (2015).

[27] L. Bernard, L. Blanchet, A. Bohé, G. Faye, and S. Marsat, Phys. Rev. D 93, 084037 (2016); 95, 044026 (2017); 96, 104043 (2017); T. Marchand, L. Bernard, L. Blanchet, and G. Faye, Phys. Rev. D 97, 044023 (2018); L. Bernard, L. Blanchet, G. Faye, and T. Marchand, Phys. Rev. D 97, 044037 (2018).

[28] D. Bini and T. Damour, Phys. Rev. D 96, 064021 (2017).

[29] B. Bertotti, Nuovo Cimento 4, 898 (1956); R. P. I. Kerr, Nuovo Cimento 13, 469 (1959); B. Bertotti and J.F. Plebański, Ann. Phys. (N.Y.) 11, 169 (1960); M. Portilla, J. Phys. A 12, 1075 (1979); K. Westpfahl and M. Goller, Lett. Nuovo Cimento 26, 573 (1979); M. Portilla, J. Phys. A 13, 3677 (1980); L. Bel, T. Damour, N. Deruelle, J. Ibanez, and J. Martin, Gen. Relativ. Gravit. 13, 963 (1981); K. Westpfahl, Fortschr. Phys. 33, 417 (1985); T. Ledvinka, G. Schafer, and J. Bicak, Phys. Rev. Lett. 100, 251101 (2008).

[30] T. Damour, Phys. Rev. D 94, 104015 (2016); 97, 044038 (2018).

[31] B. Kol and M. Smolkin, Phys. Rev. D 77, 064033 (2008); Classical Quantum Gravity 25, 145011 (2008); J. B. Gilmore and A. Ross, Phys. Rev. D 78, 124021 (2008); B. Kol, M. Levi, and M. Smolkin, Classical Quantum Gravity 28, 145021 (2011); S. Foffa and R. Sturani, Phys. Rev. D 84, 044031 (2011); 87, 064011 (2013); S. Foffa, P. Mastrolia, R. Sturani, and C. Sturm, Phys. Rev. D 95, 104009 (2017); S. Foffa, P. Mastrolia, R. Sturani, C. Sturm, and W. J. T. Bobadilla, Phys. Rev. Lett. 122, 241605 (2019); J. Blümlein, A. Maier, and P. Marquard, Phys. Lett. B 800, 135100 (2020); S. Foffa and R. Sturani, Phys. Rev. D 100, 024047 (2019); S. Foffa, R. A. Porto, I. Rothstein, and R. Sturani, Phys. Rev. D 100, 024048 (2019); S. Foffa, R. Sturani, and W. J. T. Bobadilla, J. High Energy Phys. 02 (2021) 165.

[32] N. E. J. Bjerrum-Bohr, A. Cristofoli, and P. H. Damgaard, J. High Energy Phys. 08 (2020) 038.

[33] F. Cachazo and A. Guevara, J. High Energy Phys. 02 (2020) 181; N. E. J. Bjerrum-Bohr, P. H. Damgaard, G. Festuccia, L. Planté, and P. Vanhove, Phys. Rev. Lett. 121, 171601 (2018); A. Cristofoli, N. E. J. Bjerrum-Bohr, P. H. Damgaard, and P. Vanhove, Phys. Rev. D 100, 084040 (2019); P. H. Damgaard, K. Haddad, and A. Helset, J. High Energy Phys. 11 (2019) 070; A. Cristofoli, P. H. Damgaard, P. Di Vecchia, and C. Heissenberg, J. High Energy Phys. 07 (2020) 122; G. Kälin and R. A. Porto, J. High Energy Phys. 11 (2020) 106; G. Mogull, J. Plefka, and J. Steinhoff, J. High Energy Phys. 02 (2021) 048.

[34] D. A. Kosower, B. Maybee, and D. O'Connell, J. High Energy Phys. 02 (2019) 137.

[35] A. K. Collado, P. Di Vecchia, and R. Russo, Phys. Rev. D 100, 066028 (2019).

[36] G. Kälin and R. A. Porto, J. High Energy Phys. 01 (2020) 072; 02 (2020) 120.

[37] T. Damour, Phys. Rev. D 102, 024060 (2020); D. Bini, T. Damour, and A. Geralico, Phys. Rev. Lett. 123, 231104 (2019).

[38] A. Antonelli, C. Kavanagh, M. Khalil, J. Steinhoff, and J. Vines, Phys. Rev. Lett. 125, 011103 (2020); Phys. Rev. D 102, 124024 (2020). 
[39] D. Bini, T. Damour, and A. Geralico, Phys. Rev. D 102, 084047 (2020).

[40] J. Parra-Martinez, M. S. Ruf, and M. Zeng, J. High Energy Phys. 11 (2020) 023.

[41] L. Blanchet, S. Foffa, F. Larrouturou, and R. Sturani, Phys. Rev. D 101, 084045 (2020).

[42] W. Bonnor, Phil. Trans. R. Soc. A 251, 233 (1959); W. Bonnor and M. Rotenberg, Proc. R. Soc. Ser. A 289, 247 (1966); K. S. Thorne, Rev. Mod. Phys. 52, 299 (1980); L. Blanchet and T. Damour, Phys. Rev. D 37, 1410 (1988); 46, 4304 (1992); L. Blanchet and G. Schaefer, Classical Quantum Gravity 10, 2699 (1993).

[43] S. Foffa and R. Sturani, Phys. Rev. D 87, 044056 (2013); C. R. Galley, A. K. Leibovich, R. A. Porto, and A. Ross, Phys. Rev. D 93, 124010 (2016); S. Foffa and R. Sturani, Phys. Rev. D 101, 064033 (2020).

[44] M. Beneke and V. A. Smirnov, Nucl. Phys. B522, 321 (1998).

[45] A. V. Manohar and I. W. Stewart, Phys. Rev. D 76, 074002 (2007); R. A. Porto and I. Z. Rothstein, Phys. Rev. D 96, 024062 (2017).

[46] T. Damour and G. Schaefer, Nuovo Cimento B 101, 127 (1988); T. Damour, P. Jaranowski, and G. Schafer, Phys. Rev. D 62, 044024 (2000).

[47] P. V. Landshoff and J. C. Polkinghorne, Phys. Rev. 181, 1989 (1969).

[48] R. J. Glauber, in Lectures in Theoretical Physics, edited by W. E. Brittin and L. G. Dunham (Interscience Publishers, Inc., New York, 1959), Vol. I, p. 315; D. Amati, M. Ciafaloni, and G. Veneziano, Nucl. Phys. B347, 550 (1990); R. Saotome and R. Akhoury, J. High Energy Phys. 01 (2013) 123; R. Akhoury, R. Saotome, and G. Sterman, arXiv:1308.5204; P. Di Vecchia, A. Luna, S. G. Naculich, R. Russo, G. Veneziano, and C. D. White, Phys. Lett. B 798, 134927 (2019); P. Di Vecchia, S. G. Naculich, R. Russo, G. Veneziano, and C. D. White, J. High Energy Phys. 03 (2020) 173; Z. Bern, H. Ita, J. Parra-Martinez, and M. S. Ruf, Phys. Rev. Lett. 125, 031601 (2020).

[49] Z. Bern, J. Parra-Martinez, R. Roiban, M. S. Ruf, C. H. Shen, M. P. Solon, and M. Zeng (to be published).

[50] Z. Bern, J. J. M. Carrasco, H. Johansson, and D. A. Kosower, Phys. Rev. D 76, 125020 (2007).

[51] D. Kosmopoulos, arXiv:2009.00141.

[52] J. Vines, J. Steinhoff, and A. Buonanno, Phys. Rev. D 99, 064054 (2019).

[53] A. Antonelli, A. Buonanno, J. Steinhoff, M. van de Meent, and J. Vines, Phys. Rev. D 99, 104004 (2019).

[54] Z. Bern, A. Luna, R. Roiban, C. H. Shen, and M. Zeng, arXiv:2005.03071.

[55] C. Cheung and M. P. Solon, Phys. Rev. Lett. 125, 191601 (2020); G. Kälin, Z. Liu, and R. A. Porto, Phys. Rev. D 102, 124025 (2020); Z. Bern, J. Parra-Martinez, R. Roiban, E. Sawyer, and C. H. Shen, arXiv:2010.08559.

[56] P. Di Vecchia, C. Heissenberg, R. Russo, and G. Veneziano, Phys. Lett. B 811, 135924 (2020); arXiv:2101.05772.

[57] J. M. Henn, Phys. Rev. Lett. 110, 251601 (2013); J. M. Henn, A. V. Smirnov, and V. A. Smirnov, J. High Energy Phys. 03 (2014) 088.

[58] N. Wex and G. Schäfer, Classical Quantum Gravity 10, 2729 (1993).
[59] C. Cheung, N. Shah, and M. P. Solon, Phys. Rev. D 103, 024030 (2021).

[60] T. Damour, Phys. Rev. D 102, 124008 (2020).

[61] J. Blümlein, A. Maier, P. Marquard, and G. Schäfer, Nucl. Phys. B955, 115041 (2020).

[62] J. Blümlein, A. Maier, P. Marquard, and G. Schäfer, Nucl. Phys. B965, 115352 (2021).

[63] J. Blümlein, A. Maier, P. Marquard, and G. Schäfer, arXiv:2101.08630.

[64] E. Herrmann, J. Parra-Martinez, M. S. Ruf, and M. Zeng, arXiv:2101.07255.

[65] See Supplemental Material at http://link.aps.org/ supplemental/10.1103/PhysRevLett.126.171601 for the two-body Hamiltonian in Mathematica format.

[66] L. Barack, M. Colleoni, T. Damour, S. Isoyama, and N. Sago, Phys. Rev. D 100, 124015 (2019).

[67] W. D. Goldberger and A. Ross, Phys. Rev. D 81, 124015 (2010); W. D. Goldberger, A. Ross, and I. Z. Rothstein, Phys. Rev. D 89, 124033 (2014).

[68] J. Vines, Classical Quantum Gravity 35, 084002 (2018); D. Bini and T. Damour, Phys. Rev. D 96, 104038 (2017); 98, 044036 (2018); A. Guevara, A. Ochirov, and J. Vines, J. High Energy Phys. 09 (2019) 056; Phys. Rev. D 100, 104024 (2019); M. Z. Chung, Y. T. Huang, J. W. Kim, and S. Lee, J. High Energy Phys. 04 (2019) 156; B. Maybee, D. O'Connell, and J. Vines, J. High Energy Phys. 12 (2019) 156; H. Johansson and A. Ochirov, J. High Energy Phys. 09 (2019) 040; Y. F. Bautista and A. Guevara, arXiv:1908.11349; N. Siemonsen and J. Vines, Phys. Rev. D 101, 064066 (2020); R. Aoude, K. Haddad, and A. Helset, J. High Energy Phys. 05 (2020) 051; M.Z. Chung, Y.t. Huang, J. W. Kim, and S. Lee, arXiv:2003.06600; K. Haddad and A. Helset, Phys. Rev. Lett. 125, 181603 (2020).

[69] D. Bini, T. Damour, and A. Geralico, Phys. Rev. D 101, 044039 (2020); K. Haddad and A. Helset, arXiv: 2008.04920; R. Aoude, K. Haddad, and A. Helset, arXiv: 2012.05256.

[70] A. Luna, R. Monteiro, I. Nicholson, D. O'Connell, and C. D. White, J. High Energy Phys. 06 (2016) 023; W. D. Goldberger and A. K. Ridgway, Phys. Rev. D 95, 125010 (2017); W. D. Goldberger, S. G. Prabhu, and J. O. Thompson, Phys. Rev. D 96, 065009 (2017); W. D. Goldberger and A. K. Ridgway, Phys. Rev. D 97, 085019 (2018); D. Chester, Phys. Rev. D 97, 084025 (2018); W. D. Goldberger, J. Li, and S. G. Prabhu, Phys. Rev. D 97, 105018 (2018); C. H. Shen, J. High Energy Phys. 11 (2018) 162; Y. F. Bautista and A. Guevara, arXiv:1908.11349; W. D. Goldberger and J. Li, J. High Energy Phys. 02 (2020) 092; G. L. Almeida, S. Foffa, and R. Sturani, J. High Energy Phys. 11 (2020) 165.

[71] A. Laddha and A. Sen, J. High Energy Phys. 09 (2018) 105; 10 (2018) 056; Phys. Rev. D 100, 024009 (2019); B. Sahoo and A. Sen, J. High Energy Phys. 02 (2019) 086; M. Ciafaloni, D. Colferai, and G. Veneziano, Phys. Rev. D 99, 066008 (2019); A. Laddha and A. Sen, Phys. Rev. D 101, 084011 (2020); A. P. V. and A. Manu, Phys. Rev. D 101, 046014 (2020); A. P. Saha, B. Sahoo, and A. Sen, J. High Energy Phys. 06 (2020) 153; M. A. D. Ghosh, A. Laddha, and P. V. Athira, arXiv:2007.02077; B. Sahoo, J. High Energy Phys. 12 (2020) 070. 